\title{
Effect of time interval between capecitabine intake and radiotherapy on local recurrence-free survival in preoperative chemoradiation for locally advanced rectal cancer
}

\author{
Yeon Joo Kim, MD¹, Jong Hoon Kim, MD, PhD¹, Chang Sik Yu, MD, PhD², Tae Won Kim, MD, PhD³, \\ Se Jin Jang, MD, PhD ${ }^{4}$, Eun Kyung Choi, MD, PhD ${ }^{1}$, Jin Cheon Kim, MD, PhD², Wonsik Choi, MD, PhD ${ }^{5}$ \\ Departments of ${ }^{1}$ Radiation Oncology ${ }_{1}{ }^{S}$ Surgery, ${ }^{3}$ Medical Oncology, and ${ }^{4}$ Pathology, Asan Medical Center, University of Ulsan \\ College of Medicine, Seoul; ${ }^{5}$ Department of Radiation Oncology, Gangneung Asan Hospital, University of Ulsan College of \\ Medicine, Gangneung, Korea
}

Purpose: The concentration of capecitabine peaks at 1-2 hours after administration. We therefore assumed that proper timing of capecitabine administration and radiotherapy would maximize radiosensitization and influence survival among patients with locally advanced rectal cancer.

Materials and Methods: We retrospectively reviewed 223 patients with locally advanced rectal cancer who underwent preoperative chemoradiation, followed by surgery from January 2002 to May 2006. All patients underwent pelvic radiotherapy (50 Gy/25 fractions) and received capecitabine twice daily at 12 -hour intervals $\left(1,650 \mathrm{mg} / \mathrm{m}^{2} /\right.$ day). Patients were divided into two groups according to the time interval between capecitabine intake and radiotherapy. Patients who took capecitabine 1 hour before radiotherapy were classified as Group A ( $n=109)$; all others were classified as Group B $(n=114)$.

Results: The median follow-up period was 72 months (range, 7 to 149 months). Although Group A had a significantly higher rate of good responses (44\% vs. 25\%; $p=0.005$ ), the 5 -year local recurrence-free survival rates of 93\% in Group A and 97\% in Group B did not differ significantly $(p=0.519)$. The 5 -year disease-free survival and overall survival rates were also comparable between the groups.

Conclusions: Despite the better pathological response in Group A, the time interval between capecitabine and radiotherapy administration did not have a significant effect on survivals. Further evaluations are needed to clarify the interaction of these treatment modalities.

Keywords: Capecitabine, Chemoradiotherapy, Rectal neoplasms, Survival analysis

\section{Introduction}

Treatment for rectal cancer was associated with a high local failure rate before the introduction of radiotherapy (RT), chemotherapy, and total mesorectal excision (TME). However, considerable efforts to combine peri-operative RT with a 5-fluorouracil (5-FU)-based regimen have improved local control among patients with locally advanced rectal cancer,

Received 02 March 2017, Revised 20 March 2017, Accepted 28 March 2017.

Correspondence: Jong Hoon Kim, MD, PhD, Department of Radiation Oncology, Asan Medical Center, University of Ulsan College of Medicine, 88 Olympic-ro 43-gil, Songpa-gu, Seoul 05505, Republic of Korea. Tel: +82-2-3010-4434, Fax: +82-2-30106950, E-mail: jhkim2@amc.seoul.kr

(c) This is an Open Access article distributed under the terms of the Creative Commons Attribution Non-Commercial License (http://creativecommons.org/ licenses/by-nc/4.0/) which permits unrestricted non-commercial use, distribution, and reproduction in any medium, provided the original work is properly cited.

www.e-roj.org 
and further benefits were achieved with preoperative RT [14]. Notably, 5-FU has been used to treat a wide range of cancers during the past 40 years with the greatest effects against colorectal cancer, and the addition of oxaliplatin to 5-FU in adjuvant chemotherapy regimens has improved survival in colon cancer patients [5]. When the same approach was applied to the management of locally advanced rectal cancer in the German CAO/ARO/AIO-04 trial, the inclusion of oxaliplatin in preoperative chemoradiation (CRT) significantly improved the 3-year disease-free survival (DFS) [6]. However, the efficacy data from other randomized trials were inconclusive, and oxaliplatin has not yet been incorporated into standard regimens $[7,8]$.

Capecitabine is a recently developed oral prodrug of 5-FU. This prodrug is absorbed through the gastrointestinal tract mucosa and converted to 5'-deoxy-5-fluorocytidine by hepatic carboxylesterase and subsequently to 5'-deoxy-5-fluorouridine by cytidine deaminase in hepatic or tumor cells. Finally, 5-FU is released by the action of thymidine phosphorylase in a conversion that occurs preferentially in malignant cells [9-11]. This tumor-preferential activation could improve treatment efficacy and decrease systemic 5-FU exposure. In addition, oral capecitabine administration is more convenient than intravenous 5-FU administration. Encouragingly, a randomized phase-3 trial demonstrated that capecitabine was not inferior to and could therefore 5-FU [12].

Intravenous 5-FU can be administered using two methods: (1) a short-course 5-FU infusion over 96 hours during the first and last weeks of a 5-6 week course of pelvic RT and (2) a protracted-course 5-FU infusion over 120 hours during weekdays or all 7 days per week. A previous study reported a significantly increased time to relapse and improved survival with protracted infusion in a postoperative CRT setting [13]. Theoretically, capecitabine can mimic the pharmacokinetics of continuous infusion 5-FU, which yielded superior outcomes when than bolus infusion.

However, the concentrations of capecitabine and its metabolites peak at 1-2 hours after administration and decrease exponentially thereafter, with plasma half-lives of $\leq 1$ hour $[14,15]$. Furthermore, capecitabine and its metabolites do not accumulate in plasma [16]. These findings suggest that the timing of RT and capecitabine administration should be calibrated to maximize the treatment effects of both modalities. A previous retrospective analysis at our center indicated better pathological responses among patients administered capecitabine 1 hour before RT [17]. In the present study, we evaluated whether the time interval between capecitabine administration and RT would also affect survival outcomes among patients with locally advanced rectal cancer.

\section{Materials and Methods}

Patients with locally advanced rectal cancer who underwent preoperative CRT between January 2002 and May 2006 were retrospectively reviewed. Those who met the following criteria were included: (1) clinical stage T3 or T4 tumors or positive regional lymph nodes (LNs) without distant metastasis, (2) preoperative CRT regimens that included capecitabine, and (3) eventual surgical treatment. Patients were divided into two groups depending on the time interval between capecitabine intake and RT: Group A included patients who took capecitabine 1 hour before RT and Group B included those who took capecitabine $\geq 2$ hours before RT. Of the 260 patients who received preoperative CRT between January 2002 and May 2006, 223 satisfied the inclusion criteria, and 109 and 114 were classified into Group A and Group B, respectively. This study was approved by the local Institutional Review Board, and the need for informed consent was waived.

Pretreatment evaluations included complete history taking, physical examination, complete blood count (CBC), serum biochemical tests, carcinoembryonic antigen (CEA) measurement, chest $\mathrm{X}$-rays, colonofiberoscopy, abdominopelvic computed tomography (CT), endorectal ultrasound (EUS) whole-body bone scans (in cases with CEA levels $>40 \mathrm{ng} / \mathrm{mL}$ ), and chest CT (in cases with CEA levels $>20 \mathrm{ng} / \mathrm{mL}$ ). Clinical staging was according to abdominopelvic CT and EUS findings, according to the American Joint Committee on Cancer tumornode-metastasis cancer staging system. Metastatic LNs were defined as those $>5 \mathrm{~mm}$ in size. During preoperative CRT, patients underwent weekly $\mathrm{CBC}$, biochemical tests and body weight measurement to monitor safety and compliances. Acute toxicities were evaluated using the National Cancer Institute Common Toxicity Criteria, version 4.0.

Planning CT images of patients in the prone position were obtained using a CT scanner with a 5-mm slice thickness. RT was delivered using three fields (posterior-anterior, two lateral fields) or four fields (anterior-posterior, posterioranterior, two lateral fields) at an energy of 6 or $15 \mathrm{MV}$ via a linear accelerator (Varian Clinac 1800; Varian Medical Systems, Palo Alto, CA, USA). The dose regimen was 46 Gy to the whole pelvis and a 4-Gy boost to the primary tumor, with a fraction size of $2 \mathrm{~Gy}$. The clinical target volume (CTV) included the primary tumor, metastatic $L N$, perirectal fat tissue, and the internal iliac and presacral LNs. The bottom of $L 5$ vertebra was 
defined as the superior CTV border, although this boundary was modulated to include gross tumors and LNs that extended beyond this limit in affected cases. The inferior border was $3 \mathrm{~cm}$ distal to the tumor. The planning target volume was defined as a 7-mm expansion of CTV in radial margins and 10$\mathrm{mm}$ expansion of CTV longitudinally.

Capecitabine was administered twice daily at a total daily dose of $1,650 \mathrm{mg} / \mathrm{m}^{2}$ beginning on day 1 of RT. From January 2002 to September 2004, patients were instructed to take capecitabine at 12-hour intervals approximately 30 minutes after breakfast and dinner. We obtained information about capecitabine intake times through telephone calls to the patients. Most patients had regular dining times and RT schedules. Beginning in October 2004, all patients were instructed to take capecitabine 1 hour before RT. Radiation oncologists educated patients regarding proper capecitabine intake during weekly monitoring, and radiation therapists also routinely confirmed capecitabine administration before every scheduled RT session.

Surgical resection was performed 6-8 weeks after preoperative CRT, using standard TME and autonomic nerve preservation procedures. All surgeries were performed by qualified colorectal surgeons who had performed >100 TME procedures each year. Four weeks after surgery, adjuvant chemotherapy of 6 cycles of capecitabine was started. One cycle consisted of $2,500 \mathrm{mg} / \mathrm{m}^{2} /$ day for 14 days, followed by a 7-day break. For pathological evaluations, primary tumors were sliced into 3-mm-thick sections that were stained with hematoxylin and eosin and subjected to microscopic examination. If tumor cells were not detected in the initial examination, thinner additional slices were taken from the residual tumor to ensure a thorough inspection. In addition, LNs were completely dissected from perirectal fat tissues and subjected to microscopic examination. Similar to our previous study, a complete response (CR) was defined as the absence of residual tumor. We also evaluated good responses which were defined as cases involving residual tumors $<5 \mathrm{~mm}$ in size. However, the retrospective nature of this study precluded the use of Dworak's classification of pathological response.

The primary end-point of the present study was 5-year local recurrence-free survival (LRFS). The secondary end-points were 5-year DFS, 5-year overall survival (OS), pathological response, and acute toxicity. Survival was calculated from the first day of preoperative CRT. Local recurrence was defined as an infield recurrence. The Kaplan-Meier method and log-rank test were used for survival analyzes. The Cox regression test was implemented in multivariate analyzes of significant factors affecting LRFS. Pearson chi-square test was used to compare pathological responses. A two-sided p-value of $<0.05$ was considered to indicate significance.

\section{Results}

The patients' characteristics are summarized in Table 1. Groups $A$ and $B$ were similar with respect to most variables, including the sphincter-saving rates ( $80 \%$ in both groups; $p=0.999$ ). However, Group B included more $T 4 \mathrm{~N}+$ patients than Group A

Table 1. Patient characteristics

\begin{tabular}{|c|c|c|c|}
\hline & $\begin{array}{c}\text { Group A } \\
(n=109)\end{array}$ & $\begin{array}{l}\text { Group B } \\
(n=114)\end{array}$ & p-value \\
\hline Age (yr) & $52(29-73)$ & $55(30-76)$ & 0.54 \\
\hline \multicolumn{4}{|l|}{ Gender } \\
\hline Male & $65(60)$ & $78(68)$ & \multirow[t]{2}{*}{0.17} \\
\hline Female & $44(40)$ & $36(32)$ & \\
\hline \multicolumn{4}{|c|}{ Histologic differentiation } \\
\hline Well & $17(16)$ & $21(18)$ & \multirow[t]{4}{*}{0.34} \\
\hline Moderately & $69(63)$ & $73(64)$ & \\
\hline Poorly & $4(4)$ & $8(7)$ & \\
\hline Others & $19(17)$ & $12(11)$ & \\
\hline \multicolumn{4}{|c|}{ Preoperative clinical stage } \\
\hline T3NO & $25(23)$ & $17(15)$ & \multirow[t]{5}{*}{0.00} \\
\hline T4NO & $0(0)$ & $4(3)$ & \\
\hline $\mathrm{T} 2 \mathrm{~N} 1$ & $1(1)$ & $0(0)$ & \\
\hline T3N1-2 & $77(71)$ & $69(61)$ & \\
\hline T4N1-2 & $6(5)$ & $24(21)$ & \\
\hline \multicolumn{4}{|c|}{ Initial tumor size (cm) } \\
\hline$<4$ & $17(16)$ & $28(25)$ & \multirow[t]{3}{*}{0.97} \\
\hline $4-6$ & $67(61)$ & $60(52)$ & \\
\hline$>6$ & $25(23)$ & $26(23)$ & \\
\hline \multicolumn{4}{|c|}{ Initial CEA (ng/mL) } \\
\hline$<20$ & $98(90)$ & $103(90)$ & \multirow[t]{2}{*}{0.95} \\
\hline$\geq 20$ & $11(10)$ & $11(10)$ & \\
\hline \multicolumn{4}{|c|}{ Lymphovascular invasion } \\
\hline$(-)$ & $103(94)$ & $96(84)$ & \multirow[t]{2}{*}{0.01} \\
\hline$(+)$ & $6(6)$ & $18(16)$ & \\
\hline \multicolumn{4}{|c|}{$\begin{array}{l}\text { Sphincter preserving } \\
\text { surgery }\end{array}$} \\
\hline Yes & $87(80)$ & $91(80)$ & \multirow[t]{2}{*}{1.00} \\
\hline No & $22(20)$ & $23(20)$ & \\
\hline \multicolumn{4}{|c|}{ Adjuvant chemotherapy } \\
\hline Yes & $102(94)$ & 107 (94) & \multirow[t]{2}{*}{0.93} \\
\hline No & $7(6)$ & $7(6)$ & \\
\hline
\end{tabular}

Values are presented as median (range) or number (\%).

Group A, patients who took capecitabine 1 hour before radiotherapy; Group B, patients who had 2 hours or more time interval between capecitabine intake and radiotherapy; CEA, carcinoembryonic antigen. 
Table 2. Compliance and $\geq$ grade 3 acute toxicities

\begin{tabular}{lcc}
\hline & $\begin{array}{c}\text { Group A } \\
(n=109)\end{array}$ & $\begin{array}{c}\text { Group B } \\
(n=114)\end{array}$ \\
\hline $\begin{array}{l}\text { Compliance } \\
\text { Radiation dose reduction }\end{array}$ & $0(0)$ & $2(2)$ \\
Capecitabine dose reduction & $4(4)$ & $7(6)$ \\
$\geq$ Grade 3 acute toxicities & & \\
Dermatitis & $0(0)$ & $2(2)$ \\
Proctitis & $1(1)$ & $0(0)$ \\
Hand-foot syndrome & $1(1)$ & $1(1)$ \\
Leukopenia & $2(2)$ & $2(2)$ \\
Anemia & $5(5)$ & $2(2)$ \\
Thrombocytopenia & $0(0)$ & $1(1)$ \\
Ileus & $7(6)$ & $7(6)$ \\
Fistula & $5(5)$ & $1(1)$ \\
Pelvic abscess & $2(2)$ & $1(1)$ \\
\hline
\end{tabular}

Values are presented as number (\%).

Group A, patients who took capecitabine 1 hour before radiotherapy; Group B, patients who had 2 hours or more time interval between capecitabine intake and radiotherapy.

( $5 \%$ vs. $21 \% ; p=0.001)$. Most patients tolerated the treatment regimen very well (Table 2). Only two patients in Group B required a radiation dose reduction, and four and seven patients in Groups A and B, respectively, required capecitabine dose reductions. The groups did not differ regarding compliance. The incidence of grade $\geq 3$ acute toxicities was similar between the groups, with 23 and 17 patients in Groups $A$ and $B$, respectively, being affected.

The median follow-up period was 72 months (range, 7 to 149 months). As shown in Fig. 1A, the groups did not differ significantly with respect to 5 -year LRFS (A vs. B, 93\% vs. 97\%; $p=0.519)$, and they had nearly identical 5 -year DFS and OS rates (Fig. 1B, 1C). We also compared survival after excluding CT4N+ patients, which comprised a higher proportion of Group B than that of Group A. Still, we observed no significant differences in survival (Fig. 2). Table 3 presents results from an analysis of several predictive factors for 5 -year LRFS. The age, pathological ypN stage, and RM status were significant in a univariate analysis. However, none was significant in a multivariate analysis.

In this study, the main failure pattern was distant metastasis. Twelve patients experienced local recurrences, and 10 also developed distant metastases (Table 4). All but one patient with ypT1NO disease exhibited poor pathological responses after preoperative CRT. The most prevalent local recurrent site was the regional $L N(n=5)$, followed by the pelvic wall $(n=4)$.

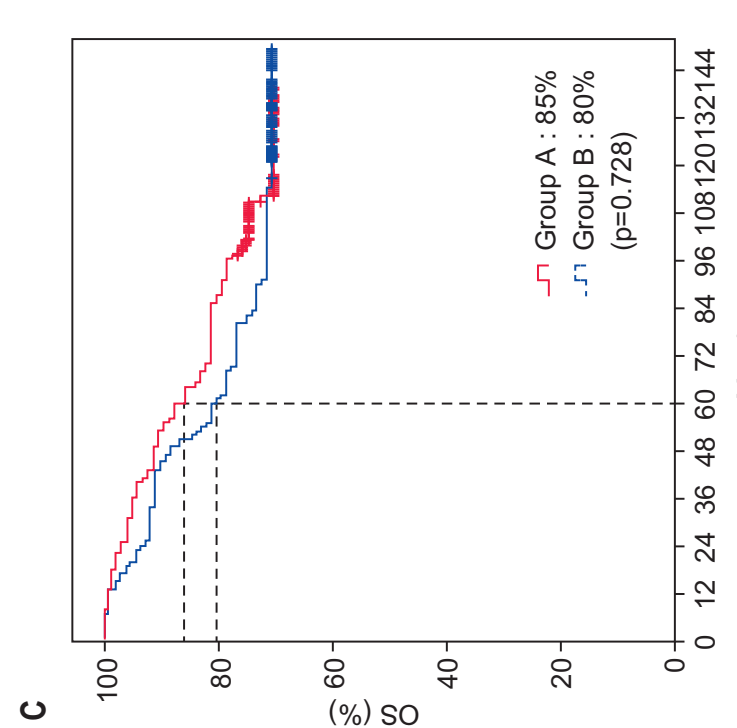

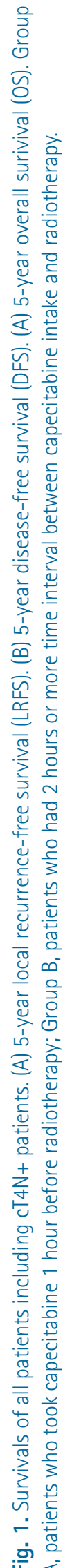

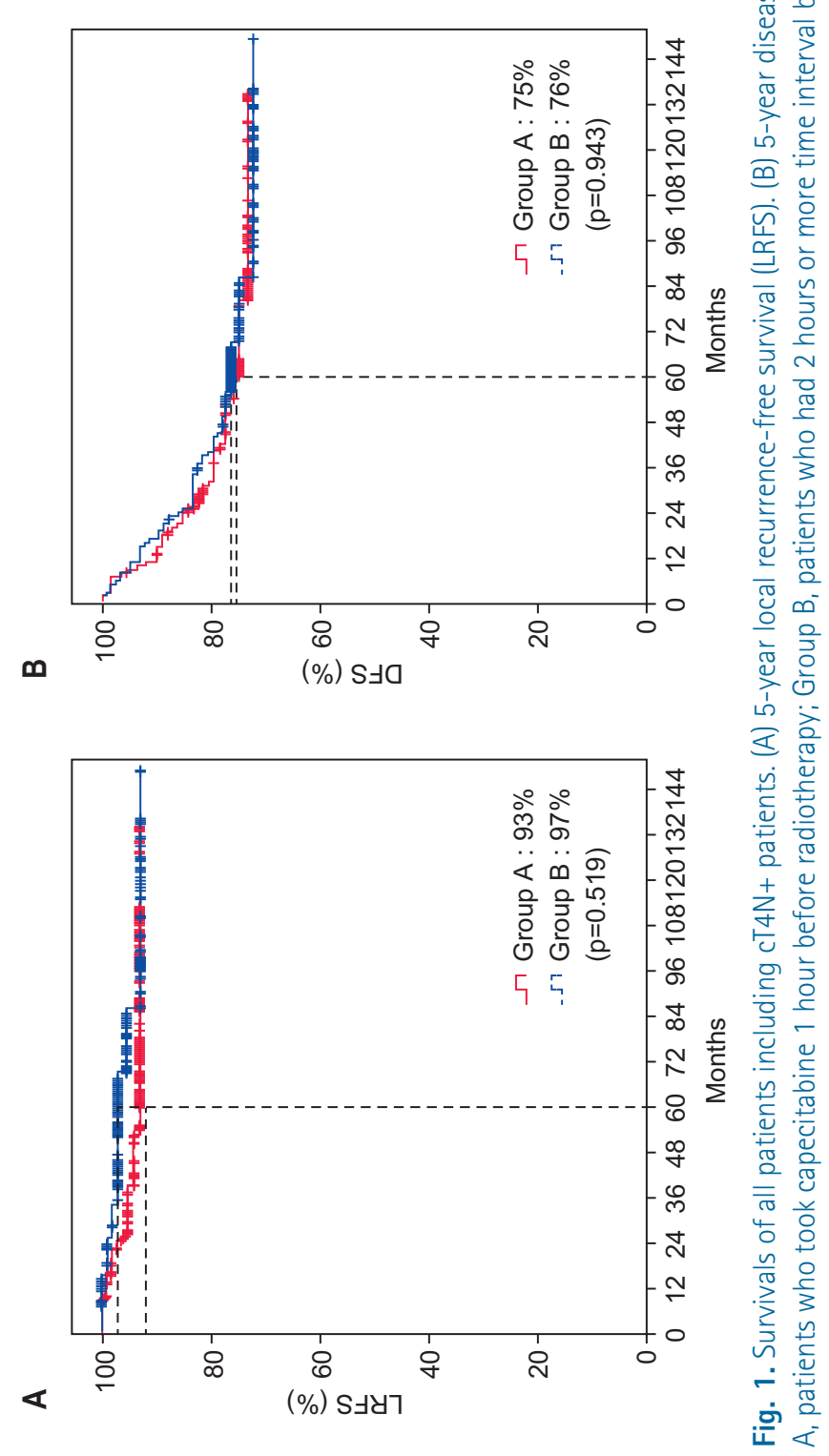

https://doi.org/10.3857/roj.2017.00010 
Table 3. Predictive factors for 5 -year LRFS

\begin{tabular}{|c|c|c|c|c|}
\hline \multirow{2}{*}{ Target volume } & \multicolumn{2}{|c|}{ Univariate } & \multicolumn{2}{|c|}{ Multivariate } \\
\hline & $5-y r$ LRFS $(\%)$ & p-value & HR $(95 \% \mathrm{Cl})$ & $p$-value \\
\hline \multicolumn{5}{|l|}{ Age (yr) } \\
\hline$<55$ & 92.2 & 0.02 & Reference & 0.86 \\
\hline$\geq 55$ & 98.1 & & $0.81(0.07-9.09)$ & \\
\hline \multicolumn{5}{|l|}{ Gender } \\
\hline Male & 96.1 & 0.13 & - & - \\
\hline Female & 93.4 & & & \\
\hline \multicolumn{5}{|l|}{ Differentiation } \\
\hline Well & 94.4 & 0.83 & - & - \\
\hline Moderate & 94.7 & & & \\
\hline Poor & 100 & & & \\
\hline Others & 96.2 & & & \\
\hline \multicolumn{5}{|l|}{ Initial tumor size $(\mathrm{cm})$} \\
\hline$<4$ & 97.1 & 0.45 & - & - \\
\hline $4-6$ & 96.0 & & & \\
\hline$>6$ & 90.8 & & & \\
\hline \multicolumn{5}{|l|}{ Initial CEA (ng/mL) } \\
\hline$<20$ & 96.1 & 0.15 & - & - \\
\hline$\geq 20$ & 85.7 & & & \\
\hline \multicolumn{5}{|c|}{ Lymphovascular invasion } \\
\hline$(-)$ & 95.6 & 0.10 & - & - \\
\hline$(+)$ & 90.5 & & & \\
\hline \multicolumn{5}{|l|}{ cT stage } \\
\hline 2 & 100 & 0.81 & - & - \\
\hline 3 & 94.3 & & & \\
\hline 4 & 94.7 & & & \\
\hline \multicolumn{5}{|l|}{ cN stage } \\
\hline 0 & 92.9 & 0.61 & - & - \\
\hline 1 & 95.6 & & & \\
\hline 2 & 100 & & & \\
\hline \multicolumn{5}{|l|}{ Response } \\
\hline Good response & 94.8 & 0.94 & - & - \\
\hline \multirow{2}{*}{\multicolumn{5}{|c|}{ ypT stage }} \\
\hline & & & & \\
\hline 0 & 100 & 0.41 & - & - \\
\hline 1 & 87.5 & & & \\
\hline 2 & 96.6 & & & \\
\hline 3 & 92.9 & & & \\
\hline 4 & 100 & & & \\
\hline \multicolumn{5}{|l|}{ ypN stage } \\
\hline 0 & 97.4 & 0.05 & Reference & 0.22 \\
\hline 1 & 87.6 & & $2.60(0.57-11.86)$ & \\
\hline 2 & 90.9 & & - & \\
\hline \multicolumn{5}{|l|}{ yp stage } \\
\hline ypCR & 100 & 0.41 & - & - \\
\hline non-ypCR & 94.2 & & & \\
\hline \multicolumn{5}{|l|}{ Resection margin } \\
\hline RO & 95.5 & 0.04 & Reference & 0.58 \\
\hline R1 & 66.7 & & $2.33(0.12-45.78)$ & \\
\hline $\mathrm{R} 2$ & 100 & & & \\
\hline \multicolumn{5}{|l|}{ Adjuvant chemotherapy } \\
\hline Yes & 95.2 & 0.72 & - & - \\
\hline No & 92.9 & & & \\
\hline
\end{tabular}

LRFS, local recurrence-free survival; $\mathrm{HR}$, hazard ratio; $\mathrm{Cl}$, confidence interval; $\mathrm{CEA}$, carcinoembryonic antigen; $C R$, complete response 


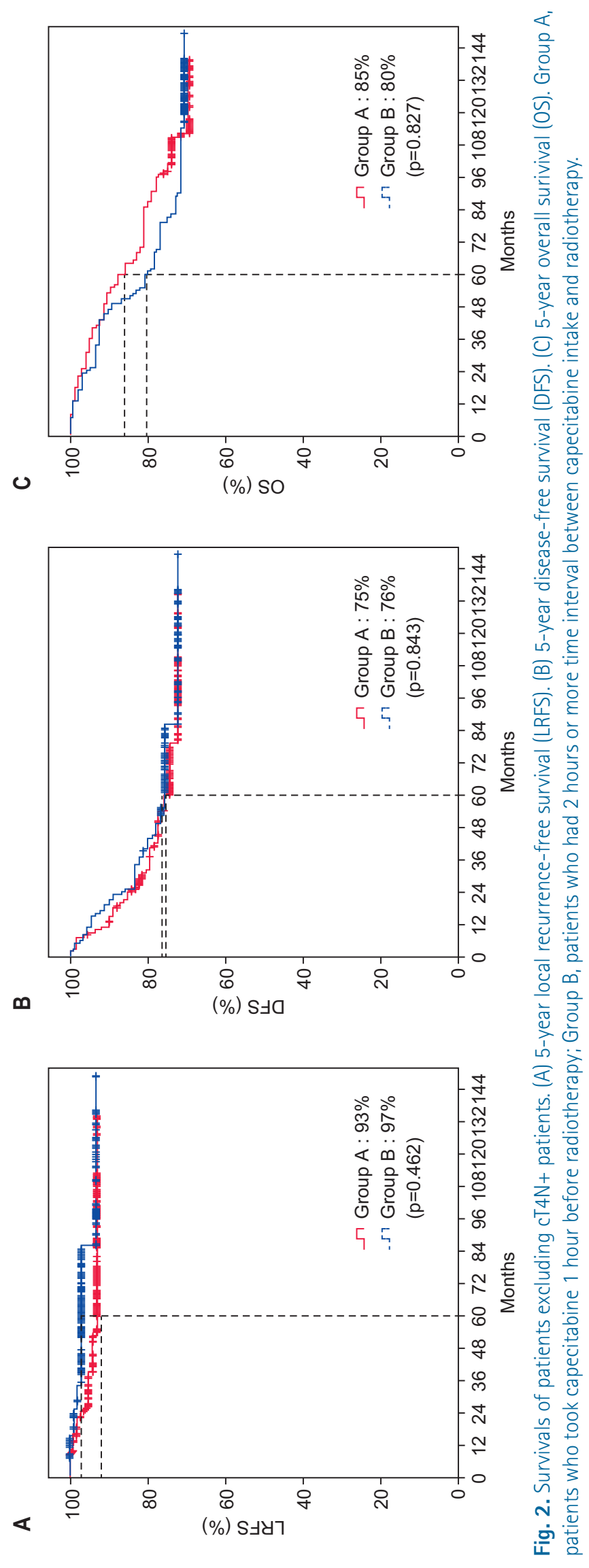

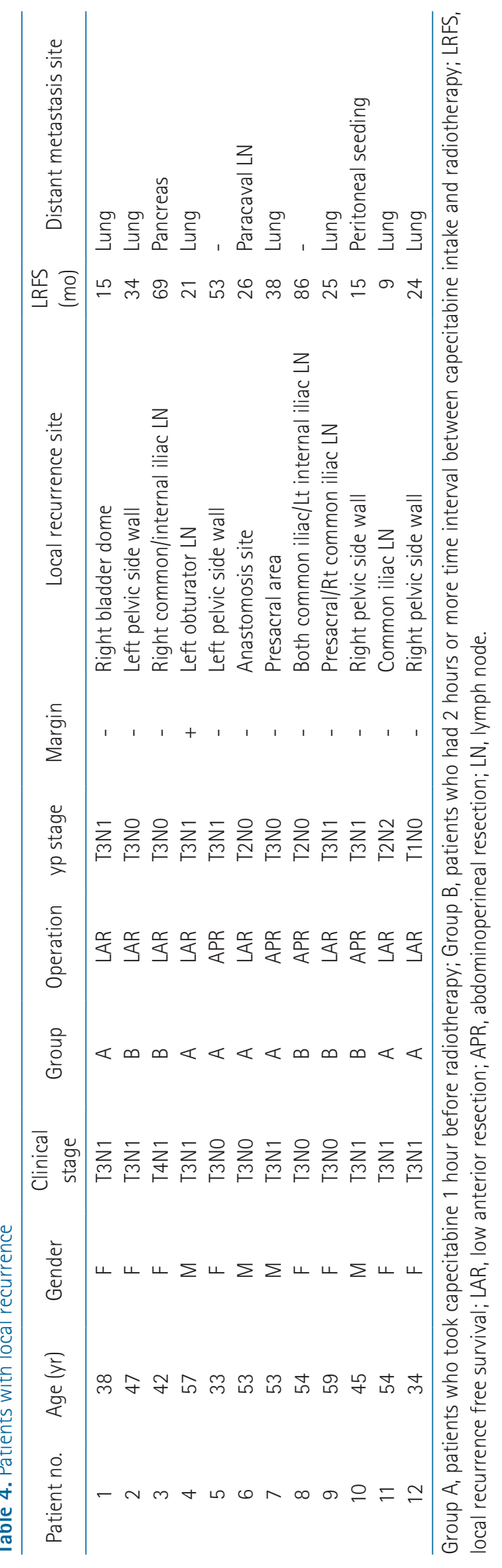

https://doi.org/10.3857/roj.2017.00010 
Although Groups A and B did not differ regarding 5-year LRFS, Group A had a significantly better pathological response. The pathological T0 rates in Groups A and B were 25\% and 9\%, respectively ( $p=0.009$ ). Group $A$ also had a significantly higher good response rate (A vs. $B, 47 \%$ vs. 24\%; $p=0.001$ ). However we observed no difference in ypN stage when only clinically node-positive patients were evaluated (A vs. B, 77\% vs. 68\%; $p=0.327)$. The improved ypTo rate also led to a significantly better ypCR rate in Group A (A vs. B, 19\% vs. 9\%; $p=0.024$ ).

\section{Discussion and Conclusion}

Many attempts have been made to improve survival rates among patients with locally advanced rectal cancer by combining chemotherapy with $\mathrm{RT}$, rather than administering preoperative RT alone. The largest trial, EORTC 22921, reported a significantly lower 5 -year local recurrence rate following preoperative RT with concurrent and/or adjuvant chemotherapy than preoperative RT alone (8\%-10\% vs. 17\%; $p=0.002$ ) [2]. In that study, the pathological To rate was also increased in the preoperative CRT arm (14\%) compared to the RT-alone arm (5\%; $p=0.004)$. The French FFCD 9203 study also reported a lower 5 -year local recurrence rate (8\% vs. $17 \% ; p<0.0001)$ and better pathological $C R$ rate (11\% vs. $4 \% ; p<0.0001)$ with preoperative CRT than RT alone [1]. In addition, both studies failed to show significant differences in 5 -year OS. Notably, the present study observed pathological CR rates of 19\% in Group A and 9\% in Group B. Maintaining a 1-hour time interval between capecitabine administration and RT appeared to maximize the radiosensitizing effect of capecitabine, thus yielding a better pathological response. However, we failed to demonstrate improvements in LRFS and $0 S$. We assume that subsequent surgical resection offset the differences in local recurrence between the groups. The time interval between treatment modalities seemed to have no effect on distant metastasis or OS. The effect of a 1-hour interval was limited to the primary rectal tumor. It may attribute to the inaccuracy of clinical nodal staging. The accuracy in detecting metastatic LNs by 3-dimensional EUS, and CT in our center were $65 \%$ and $53 \%$, respectively [18]. As result, the difference in response rates could have been reduced, and might have resulted in negative result.

Similar to our study, Bedi et al. [19] retrospectively investigated whether the timing of RT would affect the rates of pathological response and relapse in rectal cancer patients receiving capecitabine. $A$ total of 111 patients treated with preoperative CRT were instructed to take capecitabine twice daily (early morning and night). Patients were divided into the AM group, including those who underwent RT before 12 PM (closer to the morning administration of capecitabine), and the PM group, including those who underwent RT after 12 PM (several hours after morning capecitabine administration). In that study, no significant differences were observed between the AM and PM groups regarding the ypCR rate and 2-year LRFS and DFS rates. However, Bedi et al. [19] noted some limitations, including the small sample size, potential variability in the time at which each patient took capecitabine (i.e., lack of documentation), and heterogeneity in the timing of RT within the AM and PM groups. We note that our study also had some limitations. This was a retrospective analysis of a small number of patients. We investigated all available prognostic factors, however, the possibility for unknown factors which could affect the outcomes still existed. Although the time interval between capecitabine intake and RT was an important factor in the present study, time interval investigation for patients who treated between January 2002 and September 2004 was performed through telephone call, and the results could be incorrect due to the wrong answers from the patients. In addition, the initial staging did not include pelvic MRI, leading to the possibility of inaccuracies, especially when discriminating between $\mathrm{T} 3$ and $\mathrm{T} 4$ tumors.

Although we expected that the improved ypCR rate would lead to a higher sphincter-saving rate in Group $A$, this rate was $80 \%$ in both groups. We attribute this lack of difference to the low rate of patients with tumors $\leq 5 \mathrm{~cm}$ from the anal verge. Although our results should be validated by a prospective randomized trial, we cautiously suggest that 1-hour interval between capecitabine intake and RT could increase the sphincter-saving rate among patients with primary tumors near the anal verge. This practice might also improve the likelihood that patients with bulky tumors invading the mesorectal fascia will achieve a clear RM.

The present analysis did not demonstrate a significant effect of a 1-hour time interval between capecitabine administration and RT on survival among patients with locally advanced rectal cancer. However, those subjected to a 1-hour time interval exhibited better pathological responses. Further large prospective studies are warranted to clarify the interaction of capecitabine and RT.

\section{Conflict of Interest}

No potential conflict of interest relevant to this article was reported. 


\section{References}

1. Gerard JP, Conroy $T$, Bonnetain $F$, et al. Preoperative radiotherapy with or without concurrent fluorouracil and leucovorin in T3-4 rectal cancers: results of FFCD 9203. J Clin Oncol 2006;24:4620-5.

2. Bosset JF, Collette L, Calais G, et al. Chemotherapy with preoperative radiotherapy in rectal cancer. $N$ Engl J Med 2006;355:1114-23.

3. Braendengen $M$, Tveit $K M$, Berglund $A$, et al. Randomized phase III study comparing preoperative radiotherapy with chemoradiotherapy in nonresectable rectal cancer. J Clin Oncol 2008;26:3687-94.

4. Sauer R, Liersch T, Merkel $S$, et al. Preoperative versus postoperative chemoradiotherapy for locally advanced rectal cancer: results of the German CAO/ARO/AIO-94 randomized phase III trial after a median follow-up of 11 years. J Clin Oncol 2012;30:1926-33.

5. Andre $T$, Boni $C$, Navarro $M$, et al. Improved overall survival with oxaliplatin, fluorouracil, and leucovorin as adjuvant treatment in stage II or III colon cancer in the MOSAIC trial. J Clin Oncol 2009;27:3109-16.

6. Rodel C, Graeven U, Fietkau R, et al. Oxaliplatin added to fluorouracil-based preoperative chemoradiotherapy and postoperative chemotherapy of locally advanced rectal cancer (the German CAO/ARO/AIO-04 study): final results of the multicentre, open-label, randomised, phase 3 trial. Lancet Oncol 2015;16:979-89.

7. Gerard JP, Azria D, Gourgou-Bourgade S, et al. Comparison of two neoadjuvant chemoradiotherapy regimens for locally advanced rectal cancer: results of the phase III trial ACCORD 12/0405-Prodige 2. J Clin Oncol 2010;28:1638-44.

8. Aschele C, Cionini L, Lonardi S, et al. Primary tumor response to preoperative chemoradiation with or without oxaliplatin in locally advanced rectal cancer: pathologic results of the STAR01 randomized phase III trial. J Clin Oncol 2011;29:2773-80.

9. Miwa $M$, Ura $M$, Nishida $M$, et al. Design of a novel oral fluoropyrimidine carbamate, capecitabine, which generates 5-fluorouracil selectively in tumours by enzymes concentrated in human liver and cancer tissue. Eur J Cancer 1998;34:127481.

10. Ishikawa $T$, Utoh $M$, Sawada $N$, et al. Tumor selective delivery of 5-fluorouracil by capecitabine, a new oral fluoropyrimidine carbamate, in human cancer xenografts. Biochem Pharmacol 1998;55:1091-7.

11. Saif MW. Targeting cancers in the gastrointestinal tract: role of capecitabine. Onco Targets Ther 2009;2:29-41.

12. Hofheinz RD, Wenz F, Post $S$, et al. Chemoradiotherapy with capecitabine versus fluorouracil for locally advanced rectal cancer: a randomised, multicentre, non-inferiority, phase 3 trial. Lancet Oncol 2012;13:579-88.

13. O'Connell MJ, Martenson JA, Wieand HS, et al. Improving adjuvant therapy for rectal cancer by combining protractedinfusion fluorouracil with radiation therapy after curative surgery. N Engl J Med 1994;331:502-7.

14. Reigner B, Blesch K, Weidekamm E. Clinical pharmacokinetics of capecitabine. Clin Pharmacokinet 2001;40:85-104.

15. Cassidy J, Dirix L, Bissett D, et al. A Phase I study of capecitabine in combination with oral leucovorin in patients with intractable solid tumors. Clin Cancer Res. 1998;4:2755-61.

16. Mackean M, Planting $A$, Twelves $C$, et al. Phase I and pharmacologic study of intermittent twice-daily oral therapy with capecitabine in patients with advanced and/or metastatic cancer. J Clin Oncol 1998;16:2977-85.

17. Yu CS, Kim TW, Kim JH, et al. Optimal time interval between capecitabine intake and radiotherapy in preoperative chemoradiation for locally advanced rectal cancer. Int J Radiat Oncol Biol Phys 2007;67:1020-6.

18. Kim JC, Kim HC, Yu CS, et al. Efficacy of 3-dimensional endorectal ultrasonography compared with conventional ultrasonography and computed tomography in preoperative rectal cancer staging. Am J Surg 2006;192:89-97.

19. Bedi M, Das P, Skibber JM, et al. Capecitabine and timing of radiotherapy during preoperative chemoradiation for rectal cancer. Gastrointest Cancer Res 2007;1:44-8. 